\title{
Inventários florísticos na região do Caparaó Capixaba revelam novos registros para a flora do Espírito Santo
}

\section{Floristic inventories in the region of Caparaó Capixaba reveal new records for the flora of Espirito Santo}

Eduardo Alves Araújo ${ }^{1,3}$, Sustanis Horn Kunz ${ }^{1,4}$, Henrique Machado Dias ${ }^{1,4}$, Tatiana Tavares Carrijo ${ }^{2,5}$

\& João Paulo Fernandes Zorzanelli ${ }^{1,4,6}$

\begin{abstract}
Resumo
Inventários florísticos realizados ao sul do estado do Espírito Santo nos últimos anos têm auxiliado a preencher lacunas de coleta de plantas especialmente em áreas de difícil acesso, como a região do Caparaó. Estes estudos resultaram na descoberta de sete espécies de Angiospermas, de ocorrência previamente desconhecida para o estado, coletadas nas Serras do Caparaó e do Valentim. Os materiais coletados foram depositados no herbário VIES. A distribuição geográfica global foi mapeada por meio dos dados obtidos nos sítios Herbário Virtual Reflora e Flora do Brasil 2020. Os sete novos registros aqui reportados para o Espírito Santo pertencem a sete famílias diferentes de Angiospermas: Annonaceae (Guatteria pohliana), Apocynaceae (Peplonia adnata), Asteraceae (Mikania clematidifolia), Cunoniaceae (Weinmannia pinnata), Melastomataceae (Leandra quinquedentata e Pleroma foveolatum) e Ochnaceae (Ouratea campos-portoi). São fornecidos diagnoses das espécies, mapas com a distribuição global atualizada e comentários sobre os ambientes de ocorrência das espécies estudadas. Além de prover informações atualizadas ao Projeto Flora do Espírito Santo, esses dados poderão ser utilizados pelos taxonomistas para atualização do sítio Flora do Brasil 2020. Isto será especialmente útil para aquelas famílias que ainda não estão sendo monografadas na Flora do Espírito Santo. Palavras-chave: biodiversidade, conservação, Floresta Atlântica, lacunas de coleta, vegetação de altitude.
\end{abstract}

\begin{abstract}
Floristic inventories carried out in the south of the Espírito Santo state in recent years have helped to fill gaps in plant collection, especially in difficult access areas such as the Caparaó region. These studies resulted in the discovery of seven species of Angiosperms previously unknown to the state, collected in the Caparaó and Valentim highlands. The voucher specimens are housed at VIES herbarium. The global geographic distribution was obtained from the Herbário Virtual Reflora and Flora do Brasil 2020 sites. The seven new records reported for the Espírito Santo belongs to seven different families of Angiosperms: Annonaceae (Guatteria pohliana), Apocynaceae (Peplonia adnata), Asteraceae (Mikania clematidifolia), Cunoniaceae (Weinmannia pinnata), Melastomataceae (Leandra quinquedentata e Pleroma foveolatum) e Ochnaceae (Ouratea campos-portoi). Diagnoses based on species morphology, maps with updated global distribution and comments about the environment of the studied species are provided. In addition to providing up-to-date information to the Flora of Espírito Santo Project, this data can be used by taxonomists to update the Flora do Brasil 2020 site. This will be especially useful for those families who are not yet being monographed in the Flora of Espírito Santo.
\end{abstract}

Key words: biodiversity, conservation, Atlantic Forest, collection gaps, highland vegetation.

\footnotetext{
${ }^{1}$ Universidade Federal do Espírito Santo/CCAE, NUPEMASE, Av. Gov. Lindemberg 316, Centro, 299550-00, Jerônimo Monteiro, ES, Brasil.

${ }^{2}$ Universidade Federal do Espírito Santo/CCENS, Lab. Botânica, Alto Universitário, s/n, Guararema, 29500-000, Alegre, ES, Brasil.

${ }^{3}$ Universidade Federal do Espírito Santo/CCAE, Herbário VIES subcuradoria Jerônimo Monteiro, Av. Gov. Lindemberg 316, Centro, 29550-000, Jerônimo Monteiro, ES, Brasil.

${ }^{4}$ Universidade Federal do Espírito Santo/CCAE, Prog. Pós-graduação em Ciências Florestais, CCAE-UFES, Av. Gov. Lindemberg 316, 29550-000, Jerônimo Monteiro, ES, Brasil.

${ }^{5}$ Universidade Federal do Espírito Santo/CCAENS, Prop. Pós-graduação em Genética e Melhoramento, Alto Universitário, s/n, Guararema, 29500-00, Alegre, ES, Brasil.

${ }^{6}$ Autor para correspondência: jp.zorzanelli@gmail.com
} 


\section{Introdução}

A flora de diversos remanescentes do estado do Espírito Santo é pouco conhecida apesar da crescente quantidade de estudos sobre suas espécies gerados nos últimos anos, revelando novas espécies de plantas para a ciência (e.g., Kollmann \& Couto 2014; Bacci \& Goldenberg 2015; Zorzanelli et al. 2016; Sobral et al. 2017) ou ampliando o conhecimento sobre a distribuição geográfica por meio de novos registros para o estado (e.g., Favoreto \& Faria 2013; Sarnaglia Junior et al. 2014, 2015; Zorzanelli et al. 2015, 2016a). Ainda assim, o estado apresenta notórias lacunas de coleta (Dutra et al. 2015), mais expressivas em remanescentes de difícil acesso localizados em áreas montanhosas, como a região do Caparaó.

A região do Caparaó está inserida no limite norte do Complexo da Serra da Mantiqueira, que se caracteriza por possuir elevada riqueza florística (Pelissari \& Romaniuc Neto 2013), englobando áreas localizadas em diferentes municípios dos estados de Minas Gerais e Espírito Santo. A baixa representatividade de coleções para a região do Caparaó é mais expressiva para a vertente capixaba, para a qual há apenas uma listagem florística de plantas vasculares disponível na literatura (Zorzanelli et al. 2017). Os demais estudos da vegetação publicados até o presente momento para a região, foram realizados em localidades situadas no estado de Minas Gerais, por exemplo Leoni \& Chautems (2004), Mazine \& Souza (2008), Forster \& Souza (2013) e Moraes et al. (2014).

O projeto "Flora do Espírito Santo" tem como objetivo ampliar o conhecimento sobre a riqueza de espécies de plantas para o estado, por meio de monografias para grupos taxonômicos específicos e outras contribuições sobre a vegetação do Espírito Santo. O projeto também tem como objetivo fornecer suporte à pesquisadores para o desenvolvimento de monografias regionais (Dutra et al. 2015). No entanto, monografar todas as 185 famílias, 1.340 gêneros e 5.555 espécies de Angiospermas listadas para o Espírito Santo (Flora do Brasil 2020 em construção) é uma tarefa que demandará tempo e pessoal especializado. Neste intervalo, a divulgação de novos registros para táxons negligenciados permitirá ampliar o conhecimento da distribuição geográfica das espécies e auxiliar os especialistas na atualização das informações da plataforma Flora do Brasil 2020.

Considerando este cenário, este trabalho revela sete novos registros de espécies de Angiospermas de ocorrência previamente não conhecida para o Espírito Santo. Essas coletas foram realizadas em florestas montana e altomontana do Parque Nacional do Caparaó e da Serra do Valentim, ambas definidas como áreas de extrema importância biológica pelo Ministério do Meio Ambiente (MMA 2007).

\section{Material e Métodos}

Este estudo se baseou em coletas realizadas na região do Caparaó do Espírito Santo (ES), particularmente na Serra do Caparaó (2018'04'S -

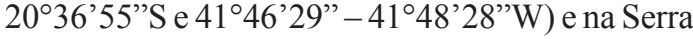

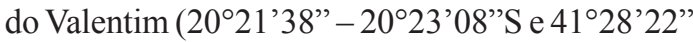
$-41^{\circ} 28^{\prime} 02^{\prime \prime W}$ ) (Fig. 1a-f), entre os anos de 2011 e 2017. Os exemplares coletados estão depositados no herbário VIES (acrônimo segundo Thiers 2017). O Parque Nacional do Caparaó (PNC) é a Unidade de Proteção Integral responsável pela conservação da fauna e da flora na Serra do Caparaó em toda a sua extensão de $31.853,12$ ha (Instituto Chico Mendes de Conservação da Biodiversidade 2015). Atividade agropecuária, extração vegetal e mineral, caça, ocupação urbana e incêndios são alguns dos principais problemas ambientais relacionados ao uso da terra destacados pelo Plano de Manejo do PNC (Instituto Chico Mendes de Conservação da Biodiversidade 2015). Já a Reserva Particular do Patrimônio Natural Toca da Onça é a única Unidade de Conservação da Serra do Valentim, protegendo 204,38 ha dos aproximadamente 4.372 ha contemplados por esse local. A Serra do Valentim é cercada por propriedades rurais que mantêm pastagens e cultivam principalmente café e Eucaliptus sp. (Zorzanelli et al. 2017).

A identificação das espécies foi realizada por meio da consulta a bibliografias especializadas (e.g., Wanderley et al. 2005; Martins et al. 2009; Morales 2010; Lobão et al. 2012), somada à consulta aos taxonomistas especialistas. Para cada espécie foi realizada uma diagnose que destaca as principais características quanto ao hábito, folhas, inflorescências e frutos, com base nos conceitos de Hickey \& King (2000). Seguido à diagnose são apresentados comentários sobre os aspectos do ambiente e da fenologia das espécies em seus locais de coleta.

A distribuição geográfica global das espécies foi consultada nas bases de dados online Flora do Brasil 2020 (em construção) e do Reflora - Herbário Virtual (<www.reflora.jbrj.gov.br>). Não foram incluídos os registros que constavam como affinis (aff.), conferatum (cf.) e de procedência duvidosa. As coordenadas utilizadas para elaboração dos 


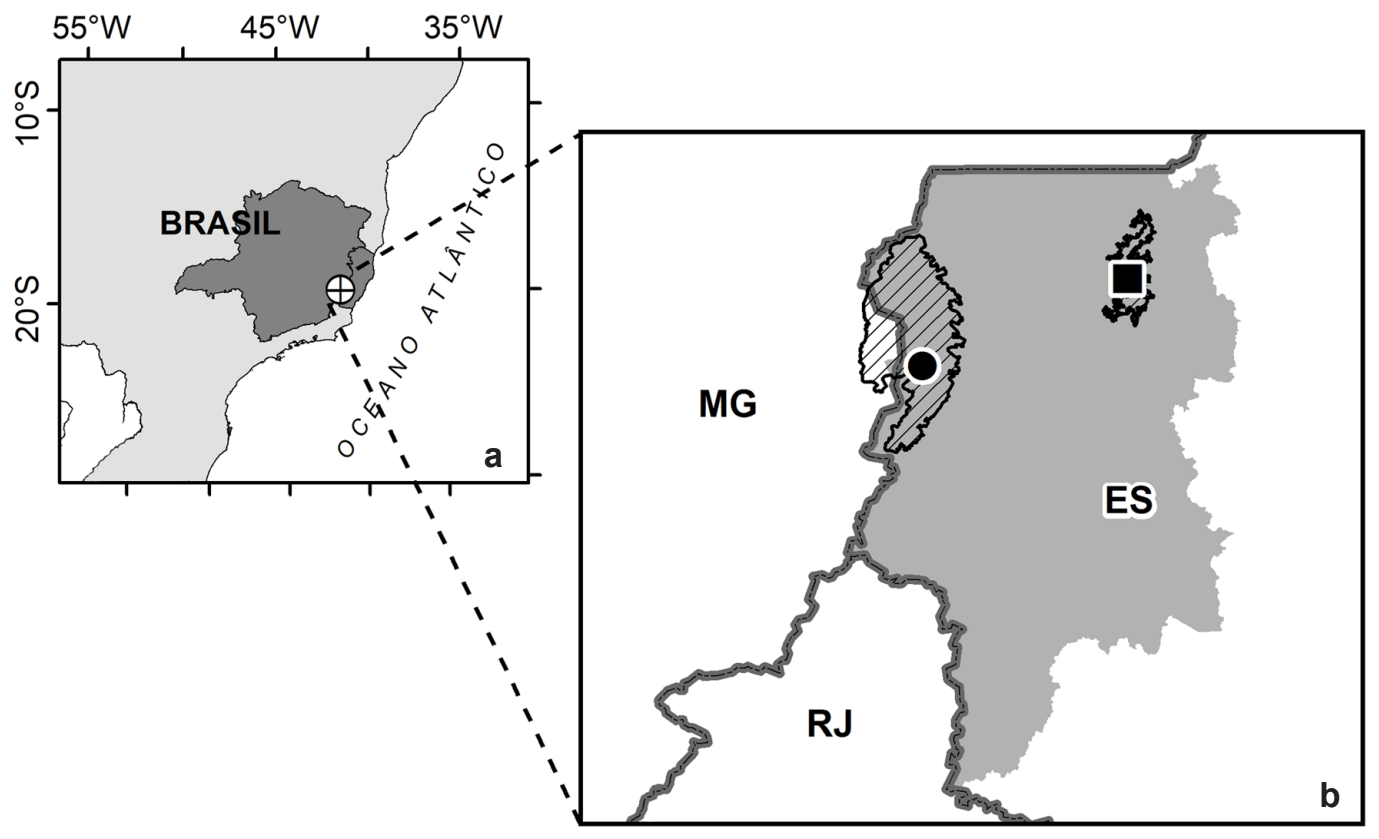

\section{SERRA DO CAPARAÓ}
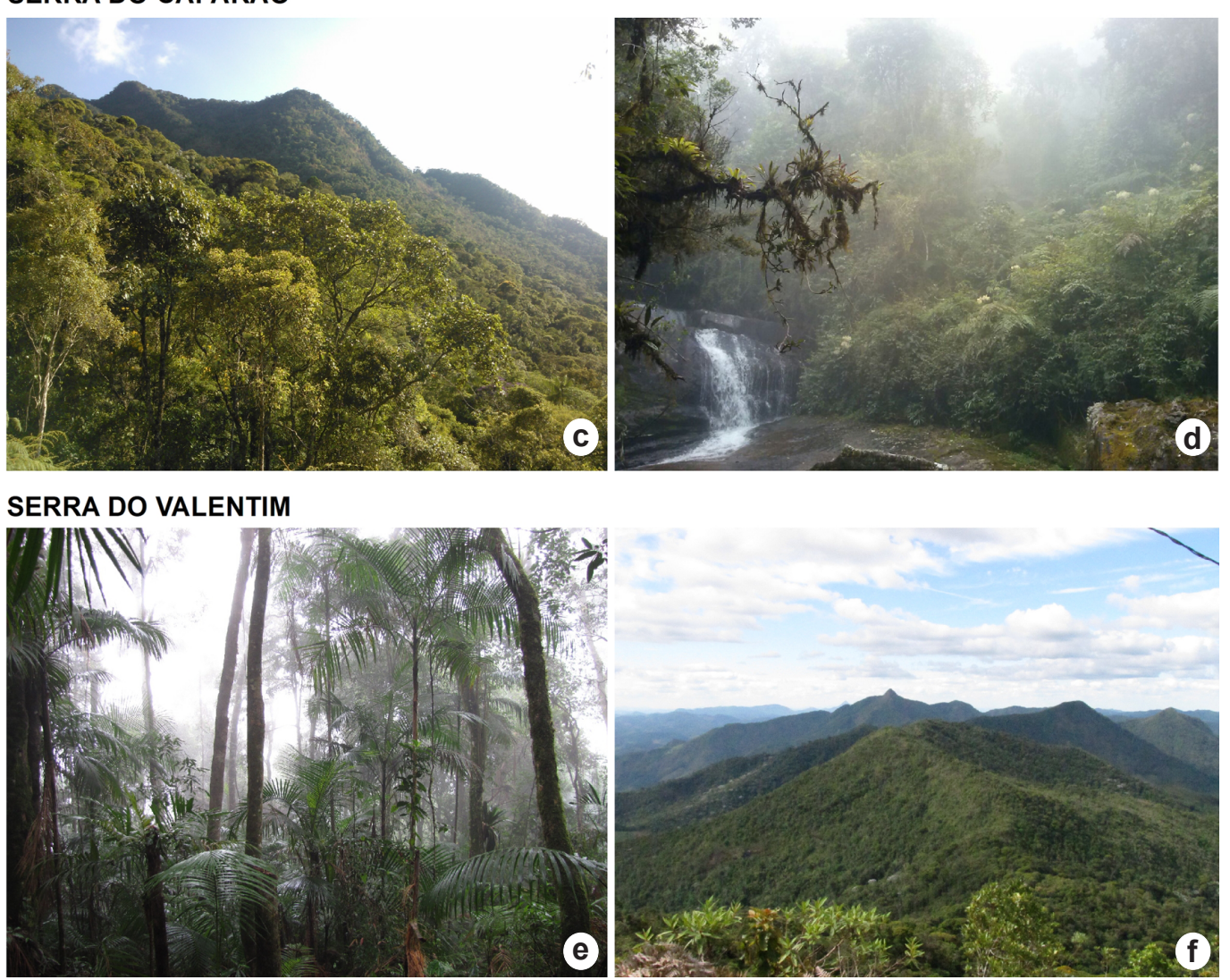

Figura 1 - Mapa indicando a localização geográfica da região do Caparaó no Estado do Espírito Santo (a) e da Serra do Caparaó (@) e da Serra do Valentim (ロ) (b). Aspectos fitofisionômicos da Serra do Caparaó (c,d) e da Serra do Valentim (e,f).

Figure 1 - Map indicating the geographical location of Caparaó region in the Espírito Santo State (a) and of Serra do Caparaó (C) and of Serra do Valentim (ם). Phytophysiognomic aspects of Serra do Caparaó (c,d) and Serra do Valentim (e,f). 
mapas de distribuição geográfica foram obtidas a partir das etiquetas das exsicatas, ou pela busca das coordenadas pelo Google Earth a partir das localidades mencionadas nas etiquetas. Os mapas foram construídos em ambiente SIG, por meio do programa ArcGis 10.1 (Environmental Systems Research Institute - ESRI 2012).

\section{Resultados e Discussão}

As sete espécies que constituem novos registros para o Espírito Santo são Guatteria pohliana Schltdl. (Annonaceae), Leandra quinquedentata (DC.) Cogn. (Melastomataceae), Mikania clematidifolia Dusén (Asteraceae), Ouratea campos-portoi Sleumer (Ochnaceae), Peplonia adnata (E.Fourn.) U.C.S.Silva \& Rapini (Apocynaceae), Pleroma foveolatum (Naudin) Triana (Melastomataceae) e Weinmannia pinnata L. (Cunoniaceae). Destas, $M$. clematidifolia está categorizada como vunerável (VU) e G. pohliana está categorizada como quase ameaçada (NT) (Martinelli \& Moraes 2013; CNC Flora 2017). As cinco demais espécies não tiveram seus status de ameaça avaliados. Dentre elas, destaca-se O. campos-portoi, conhecida por apenas quatro registros além da Serra do Valentim, todos provenientes do Parque Nacional do Itatiaia, no estado do Rio de Janeiro. Considerando os critérios de área de ocorrência e área de extensão estabelecidos pela IUCN (2012), é possível que esta espécie seja futuramente enquandrada em alguma categoria de ameaça.

A distribuição desigual de pesquisas dentro do Domínio Atlântico pode explicar, pelo menos em parte, as descontinuidades de registros de coletas de plantas no ES, comparado aos estados limítrofes. Lima et al. (2015), por exemplo, revisaram pesquisas na Floresta Atlântica desenvolvidas durante cerca de 70 anos e detectaram a existência de disparidades na distribuição destes estudos. Destaca-se ainda a ocorrência de heterogeneidade na localização dos trabalhos, com a concentração de estudos em determinadas regiões e tipos fitofisionômicos em detrimento de outros pouco estudados, como as florestas nebulares (Lima et al. 2015). A divulgação de novos registros para o ES é evidente em regiões com insuficiência de inventários florísticos, como exemplificado por Luber et al. (2016), Souza et al. (2016) e Zorzanelli et al. (2017), que listaram, respectivamente, 21, 26 e 11 novos registros para o ES. A região sul do estado, caracteriza-se pela predominância de florestas ombrófilas montanas e altomontanas, que são fitofisionomias reconhecidamente com grande incidência de espécies endêmicas, e características ambientais singulares, com grande suscetibilidade a impactos da ação antrópica (Martinelli 2007; Bruijnzeel et al. 2011).

Informações parciais sobre a distribuição geográfica de espécies dificultam a conservação da diversidade biológica (Werneck et al. 2011), e influenciam no desenho e nos resultados de estudos que dependem deste conhecimento como base, como estudos de modelagem potencial de nicho e filogeografia (e.g., Peterson 2001; Herzog et al. 2012; Guillera-Arroita et al. 2015; Roberts et al. 2016). Para efetiva conservação da biodiversidade, torna-se essencial não apenas uma base taxonômica sólida dada pela identificação acurada das espécies, mas também pelo conhecimento atualizado de sua distribuição geográfica (Versieux 2011) e ambientes preferenciais de ocorrência.

É importante destacar que a desigualdade de conhecimento florístico entre regiões, com a consequente concentração de pesquisas em um mesmo local, pode acarretar em dados de riqueza e endemismo tendenciosos (Murray-Smith et al. 2009). Áreas com alta diversidade geralmente estão localizadas próximas a centros de pesquisa botânica (Ponder et al. 2001; Werneck et al. 2011), o que pode levar a equívocos na delimitação de centros de endemismo e definição de regiões prioritárias para ações de conservação. A identificação de áreas com lacuna de conhecimento florístico é essencial para a elaboração de uma agenda de pesquisa no estado do $\mathrm{ES}$, assim como o fomento à prospecção da diversidade biológica em regiões de difícil acesso na Floresta Atlântica, como o Caparaó capixaba. Os sete novos registros de espécies para a flora do ES oriundos da região do Caparaó são apresentados a seguir.

\section{Novos registros de angiospermas para o ES}

1. Guatteria pohliana Schltdl., Linnaea 9: 321. 1834.

Fig. 2a,b

Árvore de 3,5-15,0 m alt., ramos cilíndricos. Folhas simples, alternas, margem inteira e ápice cuspidado, face abaxial com tricomas setosos esparsos, nervuras secundárias formando ângulo de $55^{\circ}$ a $90^{\circ}$ em relação à principal. Inflorescência ramiflora com flores solitárias, botões com sépalas conatas sem linha de deiscência. Carpídios elipsoides. Material examinado: Ibitirama, Santa Marta, Parque Nacional do Caparaó, parcela 06, 20²9'29”S, 41'45'27'W, 28.XI.2015, fl., E.A. Araújo 202 (VIES); 20²9'29'S, 4145'27'W, 28.XI.2015, bot. e fl., E.A. 

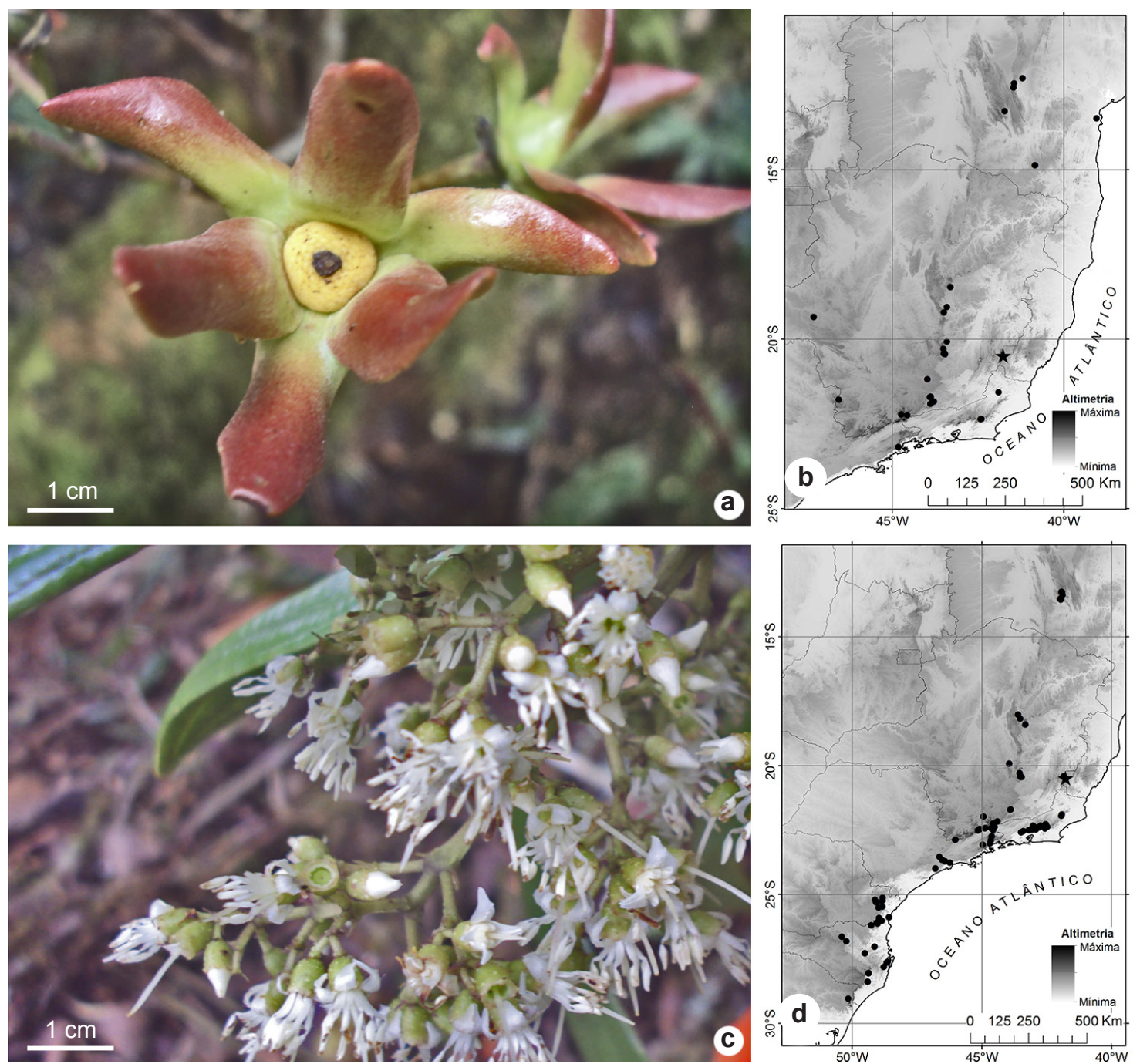

Figura 2 - Imagem e mapa de distribuição geográfica global de Guatteria pohliana (a,b) e Leandra quinquedentata $(\mathrm{c}, \mathrm{d})$. Os novos registros estão representados por uma estrela $(\star)$.

Figure 2 - Image and global geographic distribution map of Guatteria pohliana $(\mathrm{a}, \mathrm{b})$ and Leandra quinquedentata $(\mathrm{c}, \mathrm{d})$. The new records are represented by a star $(\star)$.

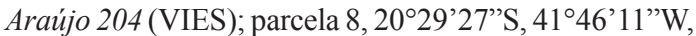
20.XII.2015, fr., E.A. Araújo 221 (VIES).

Guatteria pohliana é conhecida para os estados da Bahia, Minas Gerais, Rio de Janeiro e São Paulo (Flora do Brasil 2020 em construção). A espécie ocorre principalmente no domínio da Floresta Atlântica, em florestas ombrófila densa submontana, montana e semidecidual, já tendo sido encontrada em florestas de galeria e campo rupestre no domínio do Cerrado (Lobão \& Mello-Silva 2007; Lobão et al. 2012). No PNC, a espécie foi encontrada em floresta ombrófila densa montana e altomontana, em cota altitudinal variando entre 1.319 e $1.630 \mathrm{~m}$, sendo relativamente comum no ambiente amostrado. Coletada com flor e fruto em novembro e dezembro.

2. Leandra quinquedentata (DC.) Cogn. em Mart., Fl. bras. 19(4): 156-157. 1895. Basiônimo: Oxymeris quinquedentata DC., Prodromus Systematis Naturalis Regni Vegetabilis 3: 190. 1828.

Fig. 2c,d

Árvore 2,5-8 m alt., ramos maduros cilíndricos e jovens achatados, glabros. Folhas opostas, margens levemente onduladas, revolutas, domácias ausentes. Inflorescência paniculada, terminal. Fruto do tipo baga 5-dentado, sépalas persistentes. 
Material examinado: Ibitirama, Santa Marta, Parque

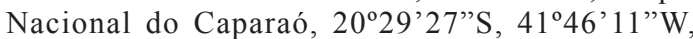
27.I.2016, fl., E.A. Araújo 256 (VIES); 20²9’27'S, 41 ${ }^{\circ} 46$ '11'W, 13.IX.2016, bot. e fr., E.A Araújo 350 (VIES); 20²9'27'S, 4146'11'W, 20.XII.2015, bot., E.A. Araújo 233 (VIES).

Leandra quinquedentata é registrada para a Bahia, Minas Gerais, Rio de Janeiro, São Paulo, Paraná, Santa Catarina e Rio Grande do Sul (Flora do Brasil 2020 em construção). A espécie foi registrada em floresta estacional semidecidual (Araújo \& Romero 2016), e em florestas ombrófila densa montana e altomontana com altitude entre 1.000 e 1.500 m (Baumgratz \& Souza 2011).
No PNC, a população de L. quinquedentata foi encontrada exclusivamente em solo com grande presença de matéria orgânica, em trecho de floresta ombrófila densa altomontana, a $1.630 \mathrm{~m}$, apresentando assim padrão de distribuição espacial agregado. Coletada com botão em setembro e com flor em dezembro e janeiro.

3. Mikania clematidifolia Dusén, Arkiv för Botanik utgivet av K. Svenska Vetenskapsakademien 9(15): 20. 1910 .

Fig. 3a,b

Liana volúvel, ramos cilíndricos. Folhas opostas, palmatífidas, segmentos lobados,
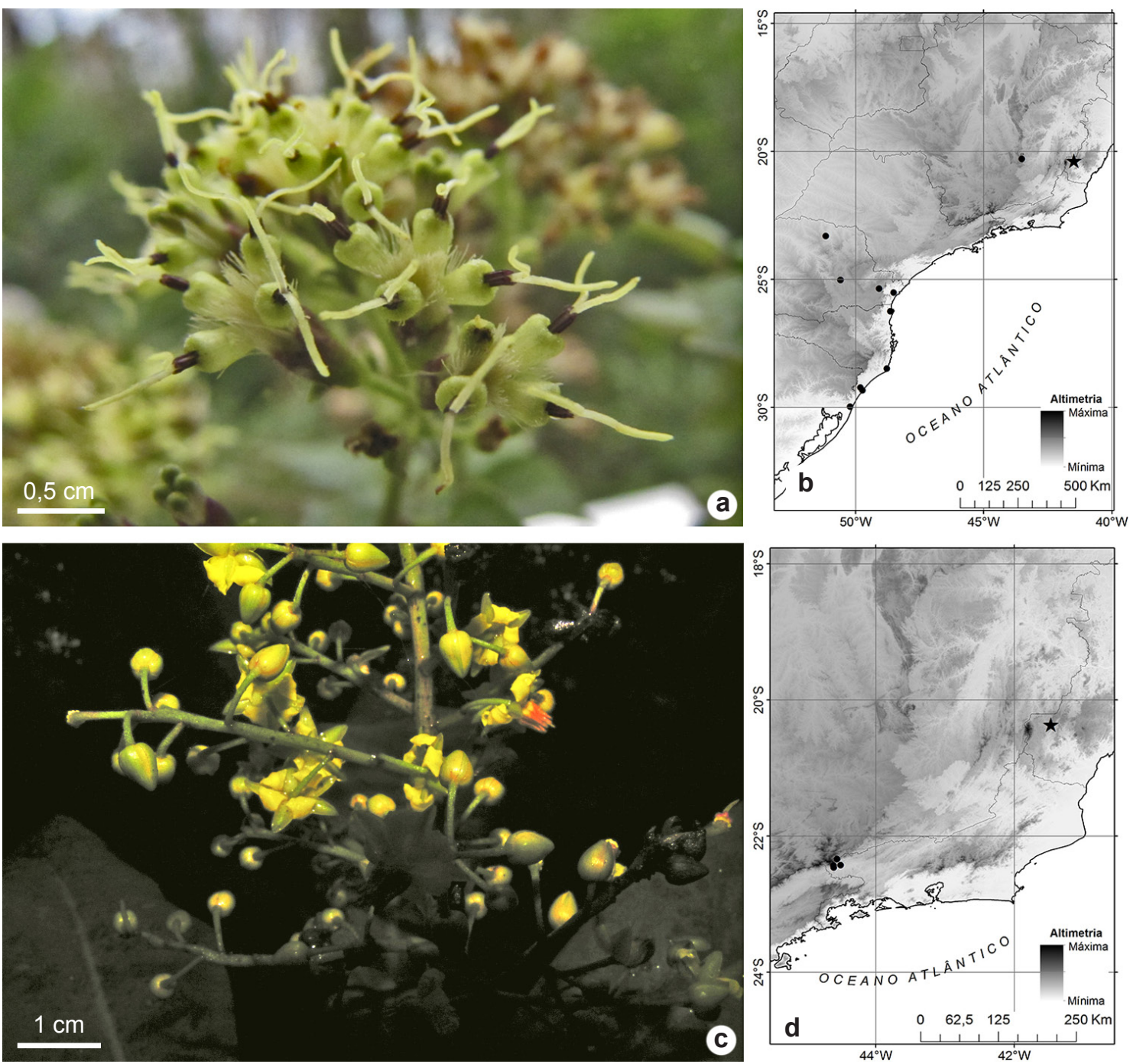

Figura 3 - Imagem e mapa de distribuição geográfica global de Mikania clematidifolia (a,b) e Ouratea campos-portoi (c,d). Os novos registros estão representados por uma estrela $(\star)$.

Figure 3 - Image and global geographic distribution map of Mikania clematidifolia $(\mathrm{a}, \mathrm{b})$ and Ouratea campos-portoi $(\mathrm{c}, \mathrm{d})$. The new records are represented by a star $(\star)$. 
face abaxial densamente coberta por tricomas glandulares, ambas as faces esparsamente cobertas por tricoma viloso. Inflorescência em capítulos paniculados vilosos. Cipselas glabras.

Material examinado: Iúna, Serra do Valentim, Floresta do Senhor Aristides, $20^{\circ} 21^{\prime} 49^{\prime \prime} \mathrm{S}, 41^{\circ} 28^{\prime} 20^{\prime \prime} \mathrm{W}$, 29.III.2016, fl., J.P.F. Zorzanelli 1491 (VIES).

Mikania clematidifolia é registrada para Minas Gerais, Paraná, Santa Catarina e Rio Grande do Sul (Flora do Brasil 2020 em construção). Habita a região litorânea e floresta estacional semidecidual no interior do Paraná (Rossetto \& Vieira 2013), e ocorre em terras baixas no litoral do Rio Grande do Sul (Ritter \& Waechter 2004). Na Serra do Valentim, M. clematidifolia habita floresta ombrófila densa montana, em local com altitude de $1.403 \mathrm{~m}$ em ambiente alterado por incêndio florestal. Coletada com flor em março. A espécie foi categorizada como "Vulnerável" (Martinelli \& Moraes 2013).

4. Ouratea campos-portoi Sleumer, Repertorium Specierum Novarum Regni Vegetabilis 42: 262. 1937.

Fig. 3c,d

Arbusto ou arvoreta $2-3,5 \mathrm{~m}$ alt., ramos cilíndricos. Folhas simples, alternas, glabras com estípulas caducas, margens levemente revolutas e crenadas. Inflorescência paniculada, terminal. Fruto esquizocarpo glabro.

Material examinado: Iúna, Serra do Valentim, trilha do Senhor Aristides, $20^{\circ} 21^{\prime} 57^{\prime \prime} \mathrm{S}, 41^{\circ} 28^{\prime} 27^{\prime}$ 'W, 30.XI.2013, bot. e fl., J.P.F. Zorzanelli 879 (VIES, RB).

Ouratea campos-portoi era registrada, até então, somente para o estado do Rio de Janeiro, no Parque Nacional do Itatiaia (Flora do Brasil 2020 em construção). Neste local, a espécie é encontrada habitando floresta ombrófila densa montana a $670 \mathrm{~m}$ de altitude. Na Serra do Valentim, O. campos-portoi também ocorre em floresta ombrófila densa montana, porém a $1.276 \mathrm{~m}$. Outros indivíduos foram avistados na mesma vegetação de forma dispersa sempre no sub-bosque da floresta. Coletada com flores em dezembro.

5. Peplonia adnata (E.Fourn.) U.C.S.Silva \& Rapini. Systematic Botany 37(3): 803. 2012. Basiônimo: Ditassa adnata E.Fourn. Mart., Fl. bras. 6(4): 256. 1885.

Fig. 4a,b

Liana, látex abundante, ramo cilíndrico. Folhas opostas, margem inteira e revoluta, ápice acuminado, face abaxial rugosa com tricomas pubérulos. Inflorescência peduncular, glabra. Flor com lobos da corola densamente cobertos por tricomas vilosos e internamente barbelados na porção basal. Fruto folículo glabro.

Material examinado: Iúna, Serra do Valentim, Floresta do Senhor Aristides, borda da vegetação, $20^{\circ} 22^{\prime} 06^{\prime \prime}$, $41^{\circ} 28^{\prime} 35^{\prime \prime} \mathrm{W}, 26 . X .2016$, fl., J.P.F. Zorzanelli 1574 (VIES).

Peplonia adnata distribui-se pelos estados de Pernambuco, Bahia, Minas Gerais, Distrito Federal, Goiás, Mato Grosso, Mato Grosso do Sul, São Paulo e Paraná (Flora do Brasil 2020 em construção). Ocorre em borda de mata ciliar, capões de mata no Cerrado, campos rupestres e Cerradão, geralmente habitando ambientes com altitude entre 520 e 1.600 m (Farinaccio 2000; Fontella-Pereira \& Ferreira 2005; Alves \& Kolbek 2009; Cavassan \& Weiser 2015). Na Serra do Valentim, a espécie foi encontrada em floresta ombrófila densa montana a $1.190 \mathrm{~m}$ de altitude, sendo observada de forma esparsa em áreas de borda. Coletada com flores em outubro.

6. Pleroma foveolatum (Naudin) Triana, em Trans. Linn. Soc. London 28: 41. $1871 . \quad$ Fig. 4c,d

Arbusto 2,5 m alt., ramos aparentemente quadrados; folhas simples, opostas, face adaxial estrigosa; inflorescência com flores solitárias, quatro bractéolas, estilete glabro, lacínias do cálice com ápice agudo; frutos cápsulas sem lacínias persistentes.

Material examinado: Ibitirama, Santa Marta, Parque Nacional do Caparaó, rio travessia P07, 20²9'34”S, 41 ${ }^{\circ} 45$ '53”'W, 27.I.2016, bot., fl. e fr., E.A Araújo 262 (VIES).

Pleroma foveolatum possui registros de ocorrência em Minas Gerais, Rio de Janeiro e São Paulo (Flora do Brasil 2020 em construção). $\mathrm{Na}$ Serra da Mantiqueira ela foi encontrada em afloramento rochoso, campos de altitude e floresta ombrófila densa altomontana (Meireles et al. 2014). $\mathrm{Na}$ porção mineira do PNC ela foi encontrada a 1.680 e $1.800 \mathrm{~m}$ de altitude, já na parte capixaba do parque ela foi coletada a $1.491 \mathrm{~m}$, em floresta ombrófila densa montana, crescendo sobre solo raso em afloramento rochoso às margens de uma cachoeira. Foi coletada com flor em janeiro.

7. Weinmannia pinnata L., Systema Nature, Tomus 2: 1005. 1759.

Fig. 5a,b

Arbustos e árvores 3-10 m alt., geralmente com troncos ramificados; folhas compostas, densamente pulverulentas ou glabrescentes, ambas as faces com folíolos pulverulentos na nervura central, raque alada, lâmina crenulada; inflorescência candelabriforme. Frutos cápsulas glabras. 

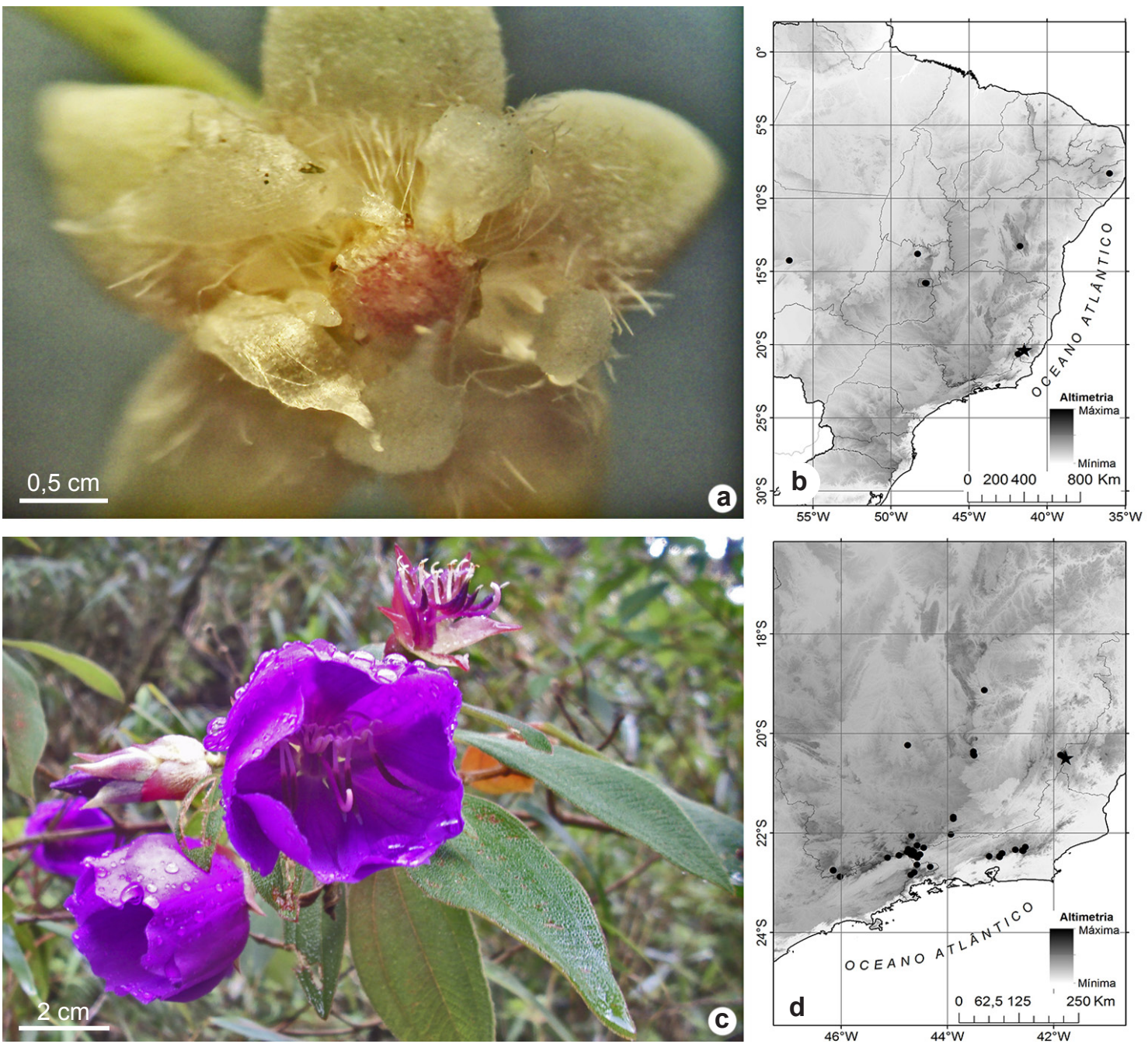

Figura 4 - Imagem e mapa de distribuição geográfica global de Peplonia adnata (a,b) e Pleroma foveolatum (c,d). Os novos registros estão representados por uma estrela $(\star)$.

Figure 4 - Image and global geographic distribution map of Peplonia adnata (a,b) and Pleroma foveolatum (c,d). The new records are represented by a $\operatorname{star}(\star)$.

Material examinado: Iúna, Serra do Valentim, Propriedade do Senhor Aristides, 20²1'53"S, 41'28'23'W, 6.XI.2011, fl., J.P.F. Zorzanelli 192 (VIES); transecto 4, 20²1'53" S, 41²8'23" W, 19.I.2012, fl. e fr., J.P.F. Zorzanelli 307 (VIES); próximo ao transecto 7, $20^{\circ} 21^{\prime} 47^{\prime}$ S, $41^{\circ} 28^{\prime} 18^{\prime \prime} \mathrm{W}, 29 . I .2016$, bot., J.P.F. Zorzanelli 1429 (VIES).

Weinmannia pinnata é encontrada nos estados do Rio de Janeiro, São Paulo, Paraná e Santa Catarina, sendo a única espécie do gênero que não é endêmica do Brasil (Flora do Brasil 2020 em construção). A espécie apresenta distribuição disjunta em relação ao Brasil, com ocorrências relatadas desde o sul do México, em países da América Central e chegando à Bolívia e Venezuela na América do Sul (Morales 2010). O gênero Weinmannia é relatado como típico de formações vegetacionais de grandes altitudes no sudeste do Brasil (Oliveira-Filho \& Fontes 2000). Ela foi amostrada na Serra do Valentim em floresta ombrófila densa montana na transição com floresta altomontana, entre 1.300 e $1.404 \mathrm{~m}$ de altitude, parecendo ter relação positiva com a elevação da altitude, já que é abundante nas porções de maior cota altitudinal. No PNC foi encontrada em formações de floresta ombrófila densa montana e altomontana, em pontos com altitude entre $1.420 \mathrm{e}$ $1.630 \mathrm{~m}$ de altitude, sendo também mais abundante 

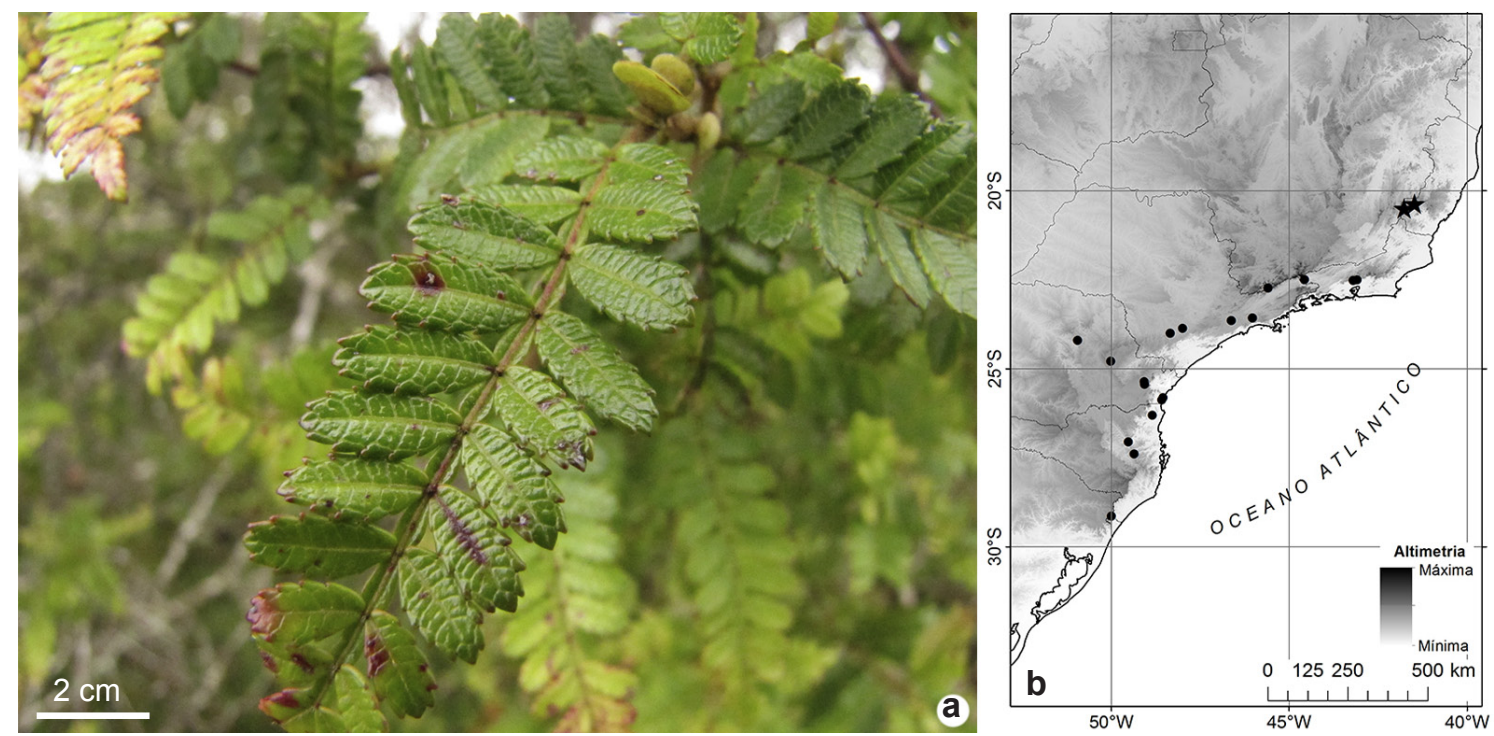

Figura 5 - Imagem e mapa de distribuição geográfica global de Weinmannia pinnata (a,b). O novo registro está representado por uma estrela $(\star)$.

Figure 5 - Image and global geographic distribution map of Weinmannia pinnata (a,b). The new record is represented by a star ( $\star$ ).

nas porções mais altas da vegetação. Coletada com flor em novembro e com fruto em janeiro.

\section{Agradecimentos}

Agradecemos aos taxonomistas Adriana Quintella Lobão (Annonaceae), Caetano Troncoso Oliveira (Asteraceae), Carmen Silvia Zickel (Cunoniaceae), Claudio Nicoletti de Fraga (Ochnaceae), Fabrício Schmitz Meyer e Marcelo Reginato (Melastomataceae), Maria Ana Farinaccio (Apocynaceae) o auxílio na identificação e confirmação dos novos registros. Às instituições de fomento CNPq e CAPES o apoio financeiro aos projetos que geraram estes resultados; ao $\mathrm{CNPq}$ a bolsa de produtividade concedida a T.T. Carrijo. Ao Sr. Plínio e família e ao Sr. Arístides e família $o$ incentivo às pesquisas na Serra do Valentim.

\section{Referências}

Alves RJV \& Kolbek J (2009) Summit vascular flora of Serra de São José, Minas Gerais, Brazil. Check List 5: 35-73.

Araújo IM \& Romero R (2016) A tribo Miconieae (Melastomataceae) no Parque Estadual do Biribiri, Diamantina, Minas Gerais, Brasil. Rodriguésia 67: 953-970

Bacci LDF \& Goldenberg R (2015) Miconia valentinensis (Melastomataceae), a new species from Espírito Santo, Brazil. Phytotaxa 195: 272.
Baumgratz JFA \& Souza MLDR (2011) Melastomataceae na Reserva Ecológica de Macaé de Cima, Nova Friburgo, Rio de Janeiro, Brasil. II - Leandra (Miconieae). Rodriguésia 62: 629-662.

Bruijnzeel LA, Mulligan M \& Scatena FN (2011) Hydrometeorology of tropical montane cloud forests: emerging patterns. Hydrological Processes 25: 465-498.

Cavassan O \& Weiser VL (2015) Vascular flora of the cerrado of Bauru-SP. Biota Neotropica 15: 1-14.

Centro Nacional de Conservação da Flora - CNC Flora. Avaliação de risco de extinção. Disponível em $<$ http://cncflora.jbrj.gov.br/portal/>. Acesso em 9 outubro 2017.

Dutra VF, Alves-Araújo A \& Carrijo TT (2015) Angiosperm checklist of Espírito Santo: using electronic tools to improve the knowledge of an Atlantic Forest biodiversity hotspot. Rodriguésia 66: 1145-1152.

ESRI - Environmental Systems Research Institute (2012). ArcGIS Desktop, version 10.2. Redlands: Environmental Systems Research Institute.

Farinaccio MA (2000) Asclepiadoideae (Apocynaceae) do Parque Nacional da Serra da Canastra, Minas Gerais, Brasil. Dissertação de Mestrado. Universidade de São Paulo, São Paulo. 92p.

Favoreto FC \& Faria APG (2013) First distribution record of Vriesea correia-araujoi E. Pereira and I. A. Penna (Bromeliaceae: Tillandsioideae) for the Espírito Santo state, southeastern Brazil. Check List 9: 81-82. 
Flora do Brasil (2020 em construção) Flora do Brasil. Jardim Botânico do Rio de Janeiro. Disponível em $<$ http://floradobrasil.jbrj.gov.br/>. Acesso em 9 outubro 2017.

Fontella-Pereira J \& Ferreira MV (2005) O gênero Macroditassa (Apocynaceae-Asclepiadoideae) no Brasil. Bonplandia 14: 7-34.

Forster W \& Souza VC (2013) Laeliinae (Orchidaceae) do Parque Nacional do Caparaó, Estados do Espírito Santo e Minas Gerais, Brasil. Hoehnea 40: 701-726.

Guillera-Arroita G, Lahoz-Monfort JJ, Elith J, Gordon A, Kujala H, Lentini PE, McCarthy MA, Tingley R \& Wintle BA (2015) Is my species distribution model fit for purpose? Matching data and models to applications. Global Ecology and Biogeography 24: 276-292.

Herzog SK, Maillard ZO, Embert D, Caballero P \& Quiroga D (2012) Range size estimates of Bolivian endemic bird species revisited: the importance of environmental data and national expert knowledge. Journal of Ornithology 153: 1189-1202.

Hickey M \& King C (2000) The Cambridge illustrated glossary of botanical terms. Cambridge University Press, Cambridge. 222p.

Instituto Chico Mendes de Conservação da Biodiversidade - ICMBio (2015) Plano de manejo para Parque Nacional do Caparaó. Agência Comunica, Brasília. $517 \mathrm{p}$.

IUCN (2012) IUCN Red List Categories and Criteria: Version 3.1. $2^{\mathrm{a}}$ ed. IUCN, Gland and Cambridge. $32 \mathrm{p}$.

Kollmann LJC \& Couto DR (2014) Pabstiella pseudotrifida L. Kollmann \&amp; D.R. Couto (Orchidaceae), a new species from Espírito Santo, Brazil. Candollea 69: 21-24.

Leoni LS \& Chautems A (2004) Flora fanerogâmica do Parque Nacional do Caparaó: Gesneriaceae. Pabstia 15: 1-11.

Lima RAF, Mori DP, Pitta G, Melito MO, Bello C, Magnago LF, Zwiener VP, Saraiva DD, Marques MCM, Oliveira AA \& Prado PI (2015) How much do we know about the endangered Atlantic Forest? Reviewing nearly 70 years of information on tree community surveys. Biodiversity and Conservation 24: 2135-2148.

Lobão AQ, Mello-Silva R \& Forzza RC (2012) Guatteria (Annonaceae) da Floresta Atlântica brasileira. Rodriguésia 63: 1039-1064.

Lobão AQ \& Mello-Silva R (2007) Guatteria (Annonaceae) do estado do Rio de Janeiro, Brasil. Rodriguésia 58: 859-884.

Luber J, Tuler AC, Leite FT, Christ JA, Guidoni-Martins KG, Zanetti M, Holunder RK, Manhães VDC, Zorzanelli JPF, Mendonça EDS, Garbin ML \& Carrijo TT (2016) List of angiosperm species in an Atlantic Forest fragment reveals collection gaps in Espírito Santo state, Brazil. Check List 12: 1835.
Martinelli G (2007) Mountain biodiversity in Brazil. Revista Brasileira de Botânica 30: 587-597.

Martinelli G \& Moraes MA(eds.) (2013) Livro vermelho da flora do Brasil. Andrea Jakobsson, Rio de Janeiro. 1100 p.

Martins SE, Wanderley MGL, Shepherd GJ, Giulietti AM \& Melhem TS (eds.) (2009) Flora fanerogâmica do estado de São Paulo. Instituto de Botânica, FAPESP, São Paulo. Vol. 6, 296p.

Mazine FF \& Souza VC (2008) Myrtaceae dos campos de altitude do Parque Nacional do Caparaó - Espírito Santo/Minas Gerais, Brasil. Rodriguésia 59: 57-74.

Meireles LD, Kinoshita LS \& Shepherd GJ (2014) Composição florística da vegetação altimontana do distrito de Monte Verde (Camanducaia, MG), Serra da Mantiqueira Meridional, Sudeste do Brasil. Rodriguésia 65: 831-859.

Ministério do Meio Ambiente - MMA (2007) Áreas prioritárias para a conservação, uso sustentável e repartição de benefícios da biodiversidade brasileira. MMA, Brasília, DF. 300p.

Moraes AM, Cunha GM \& Milward-de-Azevedo MA (2014) Composição florística de Reservas Particulares do Patrimônio Natural no município de Espera Feliz, Minas Gerais. Enciclopédia Biosfera 10: 3201-3224.

Morales JF (2010) Sinopsis del género Weinmannia (Cunoniaceae) en México y Centroamérica. Anales del Jardín Botánico de Madrid 67: 137-155.

Murray-Smith C, Brummitt NA, Oliveira-Filho AT, Bachman S, Moat J, Lughadha EMN \& Lucas EJ (2009) Plant diversity hotspots in the Atlantic Coastal Forests of Brazil. Conservation Biology 23: 151-163.

Oliveira-Filho AT \& Fontes MAL (2000) Patterns of floristic differentiation among atlantic forests in southeastern brazil and the influence of climate. Biotropica 32: 793-810.

Pelissari G \& Romaniuc Neto S (2013) Ficus (Moraceae) da Serra da Mantiqueira, Brasil. Rodriguésia 64: 91-111.

Peterson AT (2001) Predicting species' geographic distributions based on ecological niche modeling. The Condor 103: 599.

Ponder WF, Carter GA, Flemons P \& Chapman RR (2001) Evaluation of museum collection data for use in biodiversity assessment. Conservation Biology 15: 648-657.

Reflora - Herbário Virtual (2017) Herbário Virtual. Disponível em: <http://reflora.jbrj.gov.br/reflora/ herbarioVirtual/> Acesso em 9 outubro 2017.

Ritter MR \& Waechter JL (2004) Biogeografia do gênero Mikania Willd. (Asteraceae) no Rio Grande do Sul, Brasil. Acta Botanica Brasilica 18: 643-652.

Roberts DL, Taylor L \& Joppa LN (2016) Threatened or Data Deficient: Assessing the conservation status of poorly known species. Diversity and Distributions 22: 558-565. 
Rossetto EFS \& Vieira AOS (2013) Vascular flora of the Mata dos Godoy State Park, Londrina, Paraná, Brazil. Check List 9: 1020-1034.

Sarnaglia Junior VB, Zorzanelli JPF \& Guimarães EF (2014) First record of Piper scabrellum Yunck. from Espírito Santo state, southeastern Brazil. Check List 10: 414-416.

Sarnaglia Junior VB, Lirio EJ, Freitas J \& Guimarães EF (2015) New records of Peperomia armondii Yunck, Peperomia hispidula (Sw.) A. Dietr., and Peperomia mandioccana Miq. for the state of Espírito Santo, southeastern Brazil. Check List 11: 1580.

Sobral M, Costa IG, Souza MC \& Zorzanelli JPF (2017) Five new species and one new combination in Brazilian Myrtaceae. Phytotaxa 307: 233.

Souza WO, Machado JO, Tognella MMP \& AlvesAraújo A (2016) Checklist de angiospermas do Parque Estadual de Itaúnas, Espírito Santo, Brasil. Rodriguésia 67: 571-581.

Thiers B [continuously updated] Index herbariorum: a global directory of public herbaria and associated staff. New York Botanical Garden's virtual herbarium. Disponível em $<$ http://sweetgum.nybg. org/ih/>. Acesso em 4 Junho 2017.
Versieux LM (2011) Brazilian plants urgently needing conservation: the case of Vriesea minarum (Bromeliaceae). Phytotaxa 28: 35.

Wanderley MGL, Shepherd GJ, Melhem TS, Martins SE, Kirizawa M \& Giulietti AM (eds.) (2005) Flora fanerogâmica do estado de São Paulo. FAPESP, RiMa, São Paulo. Vol. 4, 392p.

Werneck MS, Sobral MEG, Rocha CTV, Landau EC \& Stehmann JR (2011) Distribution and Endemism of Angiosperms in the Atlantic Forest. Natureza \& Conservação 9: 188-193.

Zorzanelli JPF, Carrijo TT, Fiaschi P, Jardim JG, Santamaría-Aguillar D \& Amorim AM (2016) A first record of Freziera (Pentaphylacaceae) from the Brazilian Atlantic Forest, with the description of a new species. Systematic Botany 40: 1075-1080.

Zorzanelli JPF, Dias HM, Silva AG \& Kunz SH (2017) Vascular plant diversity in a Brazilian hotspot: floristic knowledge gaps and tools for conservation. Brazilian Journal of Botany.

Zorzanelli JPF, Carrijo TT, Dias HM \& Silva AG (2015) New records of angiosperms from Espírito Santo, Brazil. Check List 11: 1653. 